\title{
Non-Volatile Switching of Polycrystalline Barium Titanate Films Integrated in Silicon Photonic Waveguides
}

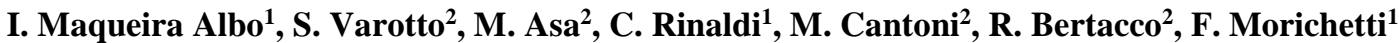 \\ 1 Dipartimento di Elettronica, Informazione e Bioingegneria Politecnico di Milano, 20133 Milano, Italy \\ 2 Dipartimento di Fisica, Politecnico di Milano, 20133 Milano, Italy \\ francesco.morichetti@polimi.it
}

\begin{abstract}
Domain switching in polycrystalline $\mathrm{BaTiO}_{3}$ is exploited to realize self-holding phase actuators in Si-photonics. A non-volatile change of the $\mathrm{BaTiO}_{3}$ refractive-index is achieved and poly- $\mathrm{BaTiO}_{3}$-coated silicon photonic circuits are demonstrated.

OCIS codes: (230.7370) Waveguides (250.5300) Photonic integrated circuits, (130.3130) Integrated optics materials, (130.2260) Ferroelectrics
\end{abstract}

\section{Introduction}

In photonic integrated circuits (PICs), low-energy optical actuators are needed to either compensate fabrication tolerances or dynamically reconfigure the circuit functionality. Thermal actuators are a reliable and widely used approach, yet requiring high power consumption and suffering from large crosstalk that limit PIC scalability. Nonvolatile actuators capable to maintain the switching state without an applied control signal are a promising route to tackle this issue, and several approaches based on phase change materials (GST) [1] and insulator-metal phase transition materials [2] have been proposed to realize intensity actuators in optical waveguides.

In this work, we investigate a different approach to realize self-holding phase actuators integrated in $\mathrm{Si}$ waveguides exploiting the ferroelectric response of polycrystalline $\mathrm{BaTiO}_{3}$ (poly-BTO). We observed a large, nonvolatile change of the poly-BTO refractive index, in the order of $10^{-2}$, which is consistent with a $90^{\circ}$ reorientation of the ferroelectric domains. We also integrated thin poly-BTO films on $\mathrm{Si}$ waveguides with a low additional propagation loss $(<1 \mathrm{~dB} / \mathrm{mm})$, enabling the realization of PICs with the proposed poly-BTO Si-photonics platform.

\section{Non-volatile optical switching in BTO thin films}

Ferroelectric domains in BTO are characterized by their in-plane ( $a$-axis) or out-of-plane ( $c$-axis) orientation. In a poly-BTO film, both phases can coexist with a random distribution. Domain re-orientation/alignment can be driven by the ferroelectric switching of the material, i.e. applying external electric fields in the direction perpendicular to the film plane. The re-orientation is expected to result in a large change of the refractive index $n$, and thanks to the remanence of ferroelectric materials, the variation is maintained when the electric field is turned off (see Fig 1.a). BTO films $(\sim 100 \mathrm{~nm})$ were grown by pulsed laser deposition (PLD) on conductive Si(001) substrates, with control of both temperature $\left(400{ }^{\circ} \mathrm{C}-600{ }^{\circ} \mathrm{C}\right)$ and oxygen pressure $\left(p_{\mathrm{O}_{2}}=20 \mathrm{~m}-80 \mathrm{mTorr}\right)$. Native $\mathrm{SiO}_{2}$ on the surface of $\mathrm{Si}$ substrates was removed by standard wet etching in hydrofluoric acid prior insertion in vacuum. The low resistivity of the Si substrate $(\sim 0.1 \Omega \cdot \mathrm{cm})$ allows ferroelectric characterization and the application of electric fields across the final heterostructure. To avoid $\mathrm{SiO}_{2}$ formation at the $\mathrm{Si}$ substrate interface, detrimental for crystalline growth of the epilayer, the first two layers of BTO were deposited at $600{ }^{\circ} \mathrm{C}$ in high-vacuum without introducing $\mathrm{O}_{2}$ in the

(a)
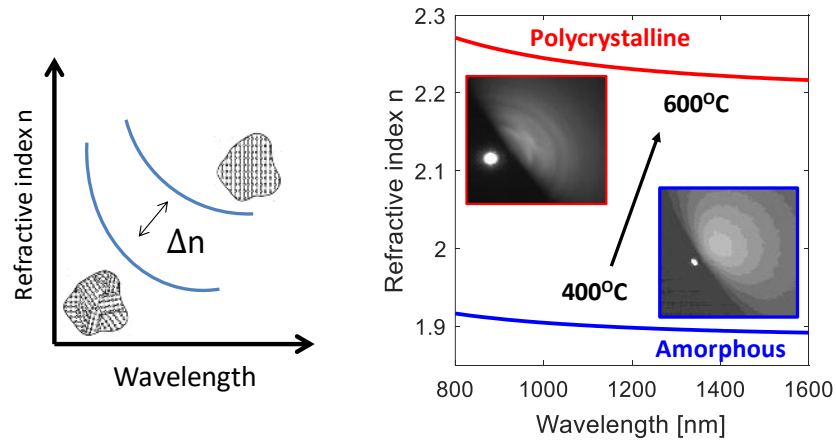

(c)

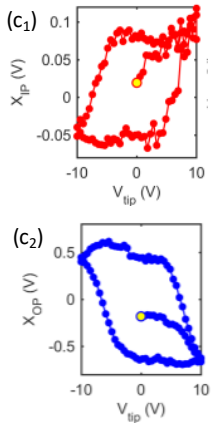

(d)

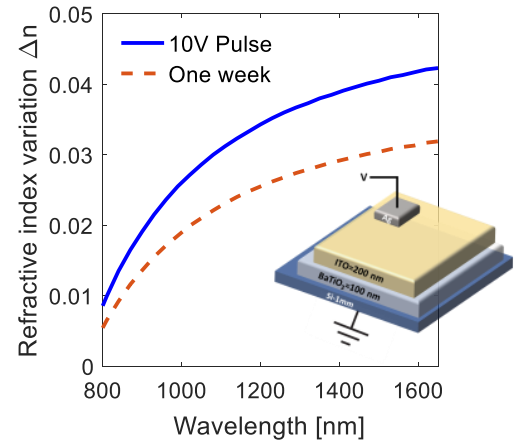

Fig. 1. (a) Schematic of the refractive index change awith domain switching in poly-BTO film. (b) Measured refractive index of amorphous and polycrystalline BTO films deposited by PLD at different temperature. RHEED measurements in the insets confirm the amorphous and polycrystalline nature of the deposited films (c) In-plane $\left(\mathrm{c}_{1}\right)$ and out-of-plane $\left(\mathrm{c}_{2}\right)$ piezoresponse spectroscopy measurement of a poly-BTO film. (d) Measured change of the refractive index of a poly-BTO film after the application of a $10 \mathrm{~V}$ pulse (solid blue line) and after 1 week relaxation (red dashed line). 

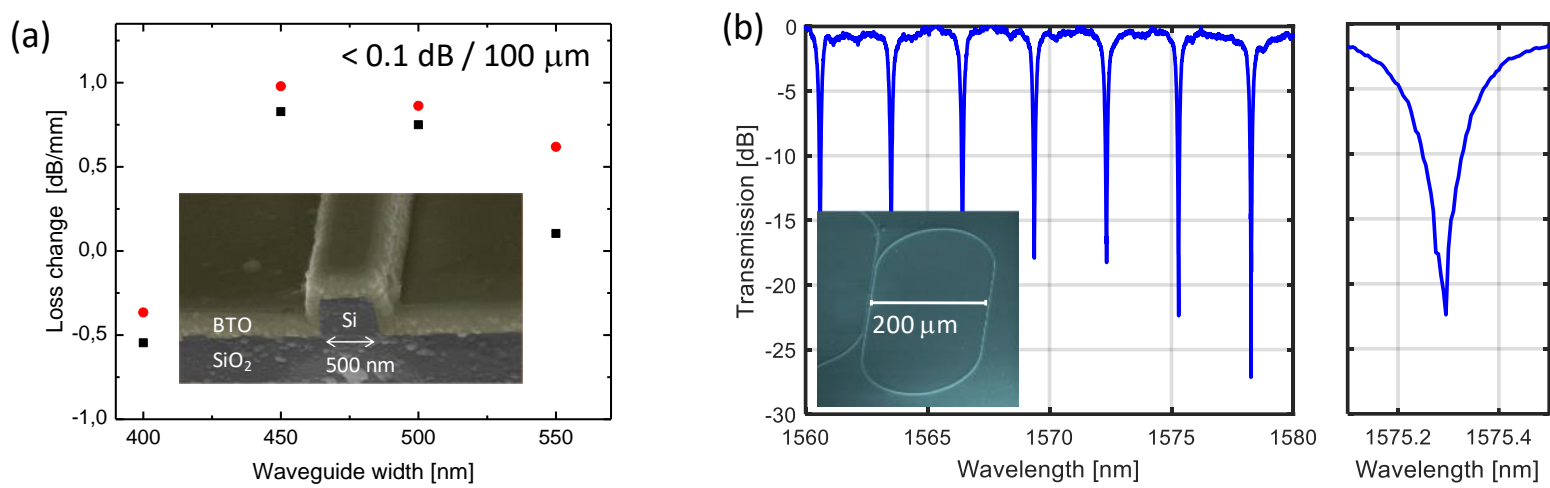

Fig. 2. (a) Variation of the propagation loss of a Si nanowaveguide (SEM cross-sectional picture in the inset) after the deposition of a 200 $\mathrm{nm}$ thick poly-BTO coating. (b) Normalized transmission of a racetrack resonator realized with a BTO-coated Si waveguide.

chamber. A post-annealing at $600^{\circ} \mathrm{C}$ was performed with a 0.5 atm $\mathrm{O}_{2}$ pressure to fill the oxygen vacancies. Reflection high-energy electron diffraction (RHEED) measurements (see insets of Fig. 1b) and X-Ray diffraction (data not shown) indicate that films deposited at $600^{\circ} \mathrm{C}$ exhibit a polycrystalline structure (poly-BTO), while lower deposition temperatures favor amorphous films (a-BTO). The surface roughness of poly-BTO films, as measured by atomic force microscopy (AFM), is between 0.7-0.9 $\mathrm{nm}$ rms. In agreement with previous reports [3], the refractive index $n$ of a-BTO films $(\sim 1.9$ at $\lambda>1300 \mathrm{~nm})$ is lower than poly-BTO films $(\sim 2.2$ at $\lambda>1300 \mathrm{~nm})$, as measured by spectroscopic ellipsometry (see Fig. 1b). By using piezoforce microscopy (PFM) we checked the ferroelectric properties of poly-BTO films, observing a significant PFM response for both in-plane (horizontal PFM, Fig. 1 $\mathrm{c}_{1}$ ) and out-of-plane components (vertical PFM, $\mathrm{c}_{2}$ ).

To perform switching experiments a $200 \mathrm{~nm}$ layer of indium tin oxide (ITO) was sputtered onto the poly-BTO film to realize the top electrode. Figure $1 \mathrm{~d}$ shows that after the application of a $10 \mathrm{~V}$ pulse, a large change of the refractive index of poly-BTO is induced (solid blue curve), that is as large as $4 \cdot 10^{-2}$ in the $1550 \mathrm{~nm}$ range. Such a significant variation suggests some degree of ferroelectric domains reorientation (from $a$ to $c$ axis) in the poly-BTO film [4], with no change in the material loss. After one week, only a small relaxation in the refractive index was observed (red dashed curve), demonstrating that the switched state was mostly preserved. We also observed that the pristine state of the film can be restored by heating the sample above the Curie temperature of BTO $\left(120^{\circ} \mathrm{C}\right)$.

\section{Low-loss poly-BTO-coated silicon waveguides and circuits}

Polycrystalline BTO was then employed as upper cladding material of Si nanowaveguides. Figure 2(a) shows the cross-sectional SEM photograph of a $220 \mathrm{~nm}$ high x $400 \mathrm{~nm}$ wide Si waveguide covered with a 200-nm-thin polyBTO film. For this waveguide geometry, the overlap factor of the guided mode with the BTO material is about $18 \%$ for TE polarization and $28 \%$ for TM polarization. Given the large $\Delta n$ measured on poly-BTO films, this would enable a $\pi$ phase shift in a waveguide actuator of less than $100 \mu \mathrm{m}$ length. As shown in Fig. 2(a), at a wavelength of $1550 \mathrm{~nm}$, both modes exhibit a loss change of less than $1 \mathrm{~dB} / \mathrm{mm}$ after the deposition of the BTO layer, thus resulting in less than $0.1 \mathrm{~dB}$ actuator loss. The loss decrease observed in the narrowest waveguide ( $400 \mathrm{~nm}$ width) is due to the mitigation of the roughness-induced scattering loss [5]. Figure 2(b) shows the TE transmission of a racetrack resonator realized with a poly-BTO coated $\mathrm{Si}$ waveguide. The device has a free spectral range of about $2.8 \mathrm{~nm}$, a 3 $\mathrm{dB}$ bandwidth of about $30 \mathrm{GHz}$, resulting in a $\mathrm{Q}$ factor of about 7000, which is limited by the optical coupling of the resonator with the bus waveguide.

To conclude, results presented in this work demonstrate the potential of the ferroelectric switching properties of poly-BTO films to realize non-volatile phase actuators integrated in Si photonics circuits.

This work was supported by Fondazione Cariplo Project Advanced Control Technologies for Integrated Optics (ACTIO) - Rif. $2016-0881$.

[1] H. Zhang, et al, "Ultracompact Si-GST hybrid waveguides for nonvolatile light wave manipulation,” IEEE Phot. Journ.10(1), 2200110 (2018)

[2] A.Joushaghani, et al., "Sub-volt broadband hybrid plasmonic-vanadium dioxide switches, Appl. Phys. Lett. 102, 061101 (2013)

[3] Thomas, Reji, D. C. Dube, M. N. Kamalasanan, and Subhas Chandra, Optical and electrical properties of BaTiO3 thin films prepared by chemical solution deposition", Thin solid films 346 (1-2), 212, (1999)

[4] M. J. Dicken, L. A. Sweatlock, D. Pacifici, H. J. Lezec, K. Bhattacharya, and H. A. Atwater, "Electrooptic modulation in thin film barium titanate plasmonic interferometers," Nanoletters 8 (11), 4048 (2008).

[5] D. Melati, A. Melloni, and F. Morichetti, "Real photonic waveguides: guiding light through imperfections," Advances in Optics and Photonics, vol. 6, no. 2, pp. 156-224, May 2014. 